\title{
Crystallography and Morphology of Antiphase Boundary-Like Structure Induced by Martensitic Transformation in Ti-Pd Shape Memory Alloy
}

\author{
Mitsuhiro Matsuda ${ }^{1}$, Toru Hara ${ }^{2}$ and Minoru Nishida ${ }^{3}$ \\ ${ }^{1}$ Department of Materials Science and Engineering, Kumamoto University, Kumamoto 860-8555, Japan \\ ${ }^{2}$ National Institute for Materials Science, Tsukuba 305-0047, Japan \\ ${ }^{3}$ Department of Applied Science for Electronics and Materials, Kyushu University, Kasuga 816-8580, Japan
}

\begin{abstract}
An antiphase boundary (APB)-like structure in the B19 martensite of Ti-Pd alloy has been investigated by transmission electron microscopy. High-resolution and high-angle annular dark-field scanning transmission electron microscopy images demonstrate the presence of an APB-like contrast along the (001) basal plane of the B19 martensite. The contrast is not inherited from the APB with a displacement vector of the type $\boldsymbol{R}=(1 / 2)\langle 111\rangle$ in the B2 parent phase. These facts suggest that the boundary is induced by the local heterogeneity of atomic movements during the martensitic transformation. Therefore, we have newly proposed that the APB-like structure in the B19 martensite is defined as the displacive transformation-induced APB. [doi:10.2320/matertrans.MBW200715]
\end{abstract}

(Received October 16, 2007; Accepted December 17, 2007; Published February 14, 2008)

Keywords: antiphase boundary, high-angle annular dark-field scanning transmission electron microscopy, martensite, displacement vector, titanium-palladium alloy

\section{Introduction}

The boundary of domains, which is formed by an orderdisorder transformation, is the so-called antiphase boundary (APB). Several investigations have been performed on the $\mathrm{APB}$ in ordered structures such as $\mathrm{B} 2, \mathrm{~L}_{2}$, and $\mathrm{L} 1_{0}$. The present authors have recently observed an APB with a displacement vector of the type $\boldsymbol{R}=(1 / 2)\langle 111\rangle$ induced by the order-disorder transformation in the stabilized B2 parent phase of the rapidly solidified (RS) Ti-Pd melt-spun alloys of near-equiatomic compositions. ${ }^{1)}$ Although the near-equiatomic Ti-Pd bulk alloys undergo a thermoelastic martensitic transformation from the B2 to B19 structure at around $800 \mathrm{~K}$ upon cooling, ${ }^{2)}$ the origin of the stabilization of the $\mathrm{B} 2$ phase in the RS Ti-Pd alloys has not been identified. This APB is the boundary where the B2 ordered domain nucleates and grows in the primarily solidified bcc disordered phase, with the two domains eventually coming into contact with each other. In the present study, we have newly found the APBlike structure in the B19 martensite of Ti-Pd bulk alloys by transmission electron microscopy. The morphology and diffraction condition of this APB-like contrast are significantly different from those of the APB contrast in the stabilized B2 phase of RS Ti-Pd alloys.

The purpose of the present paper is to clarify the crystallography and morphology of the APB-like structure in the B19 martensite of the Ti-Pd shape memory alloy by conventional transmission electron microscopy (CTEM) and high-resolution transmission electron microscopy (HRTEM). Furthermore, in order to analyze the interface structure at the atomic level in detail, high-angle annular dark-field scanning transmission electron microscopy (HAADF-STEM) technique is applied. The advantage of HAADF-STEM for the present alloy has been confirmed in the previous paper ${ }^{1)}$ since the difference in the atomic numbers between $\mathrm{Ti}(Z=22)$ and $\mathrm{Pd}(Z=46)$ is sufficiently large to obtain the Z-contrast images. The formation mechanism of the APB-like structure is also discussed on the basis of these observations.

\section{Experimental Procedure}

Ti-50.0 at\% Pd alloy was prepared from 99.7\% $\mathrm{Ti}$ and $99.8 \%$ Pd (\% by mass) by arc melting in an argon atmosphere. The ingots were homogenized in vacuum at $1273 \mathrm{~K}$ for $36 \mathrm{ks}$. Subsequently, the samples were solution-treated in vacuum at $1273 \mathrm{~K}$ for $3.6 \mathrm{ks}$, and then quenched into ice water. The TEM specimens were electropolished using a twin jet method in an electrolyte solution consisting of $20 \%$ $\mathrm{H}_{2} \mathrm{SO}_{4}$ and $80 \% \mathrm{CH}_{3} \mathrm{OH}$ by volume at around $253 \mathrm{~K}$. CTEM and HRTEM observations were carried out with JEM2000FX and FEI-Tecnai20F microscopes, respectively, which were operated at $200 \mathrm{kV}$. The HAADF-STEM observation was performed with a HD-2300C microscope. The crystal structure of the B19 martensite is of the AuCd-type with an orthorhombic cell. The lattice correspondence between the B2 parent phase and the B19 martensite is illustrated in Fig. 1. ${ }^{3)}$ The dashed and solid lines indicate B2 and B19 structures, respectively. The following lattice parameters were used for the analysis: $a_{\mathrm{B} 19}=0.489 \mathrm{~nm}$, $b_{\mathrm{B} 19}=0.281 \mathrm{~nm}$, and $\left.c_{\mathrm{B} 19}=0.456 \mathrm{~nm}^{4}\right)$

\section{Results and Discussion}

Figures 2(a) and (b) show the typical bright field image and the corresponding electron diffraction pattern of the Ti-50.0 at\% Pd alloy, respectively. The pattern in Fig. 2(b) consists of two sets of reflections from the $[1 \overline{1} 0]_{\mathrm{B} 19}$ zone axis that are in mirror symmetry with respect to the (111) plane. The traces of alternating platelets in Fig. 2(a) are parallel to the (111) plane. This fact indicates that the two sets of reflections show a (111) twin pattern, and the platelets are a (111) type I twin, which is a lattice invariant shear of the martensitic transformation from the B2 to the B19 structures in the Ti-Pd alloy. This observation is consistent with the results provided in the previous report. ${ }^{5)}$ It is notable that many line contrasts are observed along the basal plane within the twin platelets, as indicated by the arrow in Fig. 2(a). In 


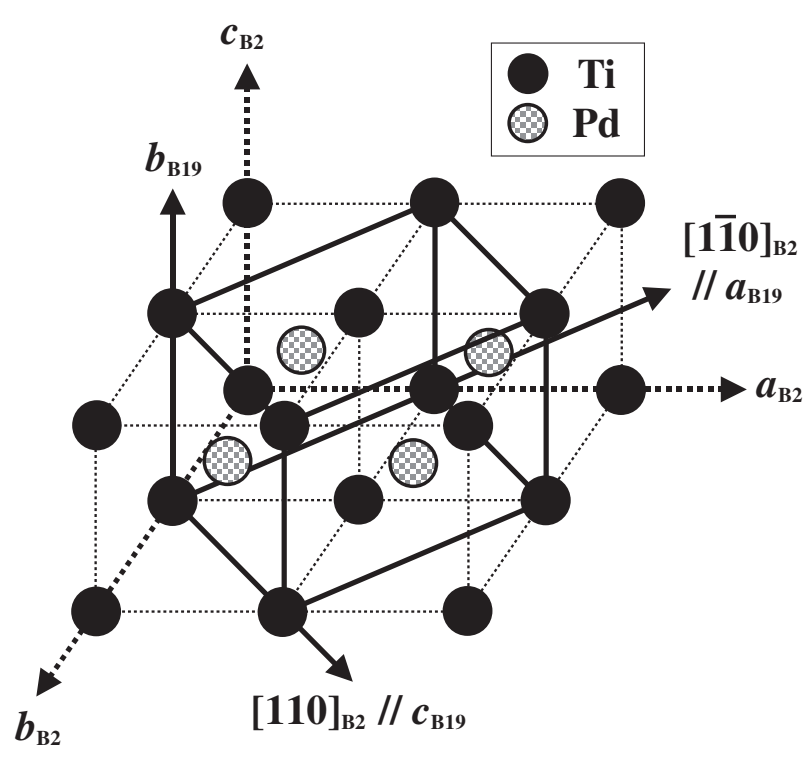

Fig. 1 Lattice correspondence between the B2 parent phase and the B19 martensite. Dashed and solid lines indicate B2 and B19 structures, respectively.

order to characterize these planar defects, CTEM observations are carried out from the $[010]_{\mathrm{B} 19}$ direction, which is another low-index zone axis parallel to the basal plane. HRTEM and HAADF-STEM observations are also performed from this zone axis as described below since the atomic columns of $\mathrm{Pd}$ and $\mathrm{Ti}$ in the B19 structure are easily distinguished, as is apparent from Fig. 1. Figures 3(a) and (b) show the bright field image and corresponding electron diffraction pattern, respectively, from the $[010]_{\mathrm{B} 19}$ zone axis. The line contrasts along the (001) basal plane of B19
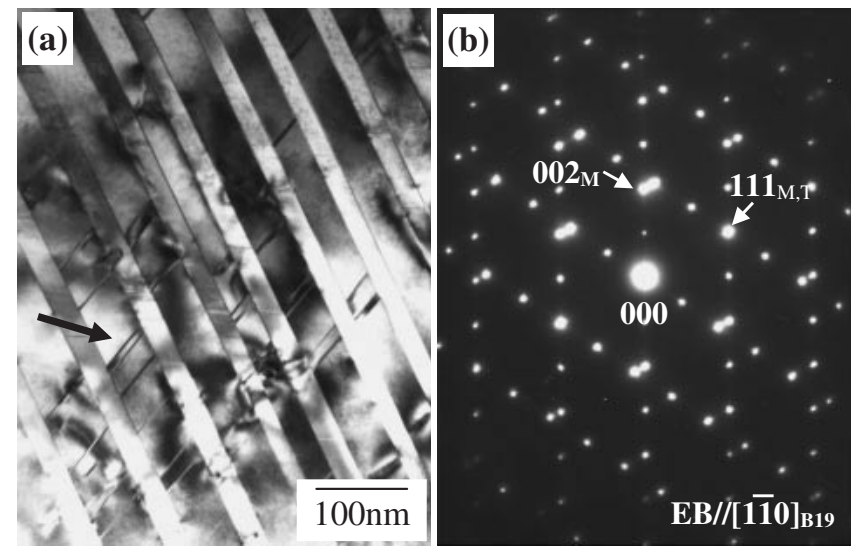

Fig. 2 (a) A Bright field image and (b) the corresponding electron diffraction pattern of a Ti-50.0 at\% Pd alloy.

martensite and curved contrasts on the non-basal plane are indicated by the single and double arrows in Fig. 3(a), respectively. Some closed loops are also observed in a large twin plate. From this point, this microstructure is defined as the APB-like structure. It can be noted that the morphology of the APB-like structure can be clearly observed in the large twin plate due to the absence of twin boundary contrast; thus, TEM observations are carried out at such regions hereafter. Figures 3(c) and (d) show the dark field images taken by using $10{ }^{*}{ }_{\mathrm{B} 19}$ and $002{ }_{\mathrm{B} 19}$ reflections, respectively. In the latter case, the $002^{*}{ }_{\mathrm{B} 19}$ reflection is used since the $001^{*}{ }_{\mathrm{B} 19}$ reflection is forbidden according to the extinction rule of the B19 structure. ${ }^{6)}$ APB-like contrasts are observed by using $10{ }^{*}{ }_{\mathrm{B} 19}$ reflection. On the other hand, no contrast is observed when using $002^{*}{ }_{\mathrm{B} 19}$ reflection. It has been widely recognized that $\pi$ contrast of the APB induced by order-disorder
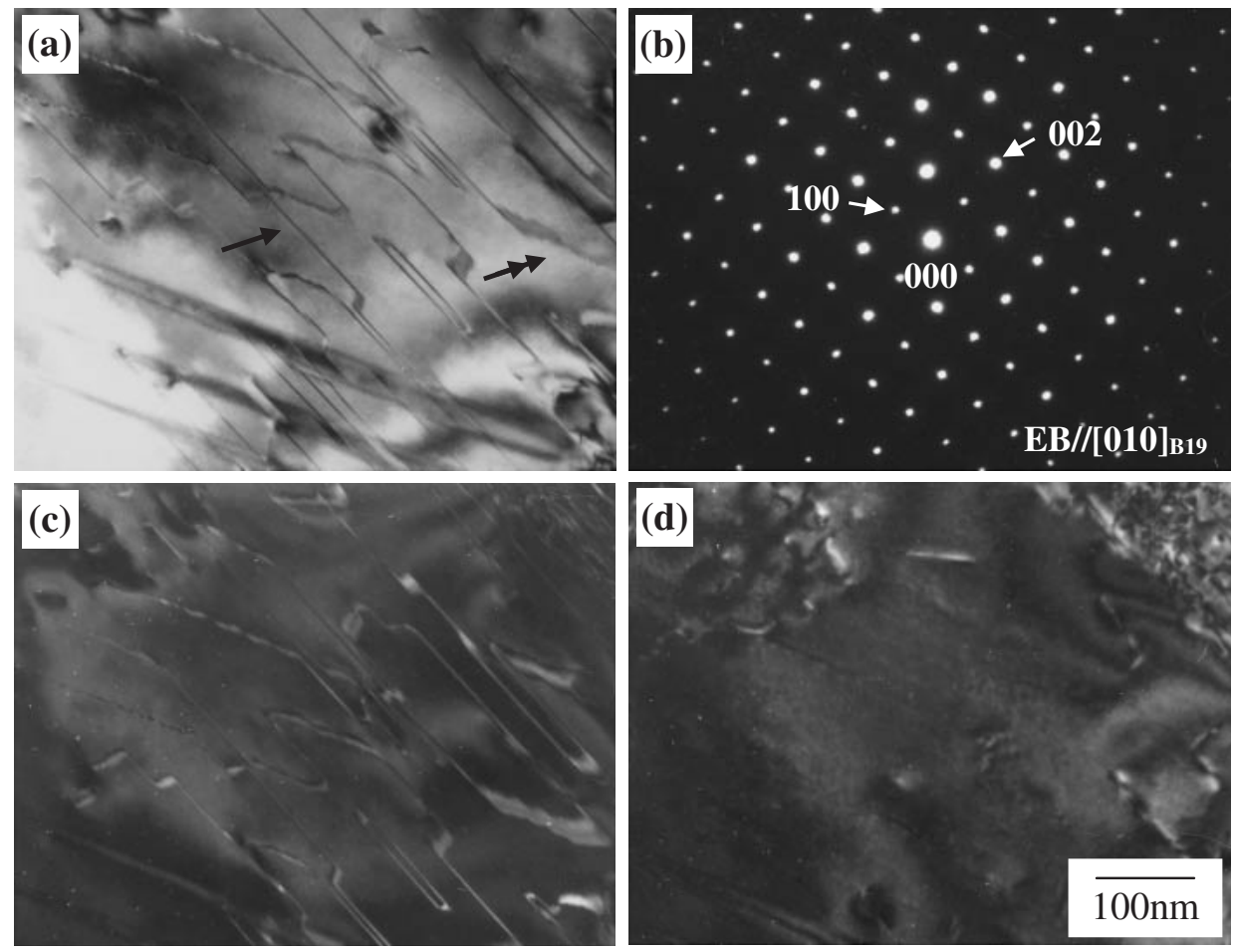

Fig. 3 (a) A Bright field image and (b) the corresponding electron diffraction pattern of a Ti-50.0 at $\%$ Pd alloy from the $[010]_{\mathrm{B} 19}$ zone axis. Dark field images of the area shown in (a). (c) APB-like structure in contrast: $g=100$ and (d) no APB-like structure in contrast: $\boldsymbol{g}=002$ with EB near [010]. 


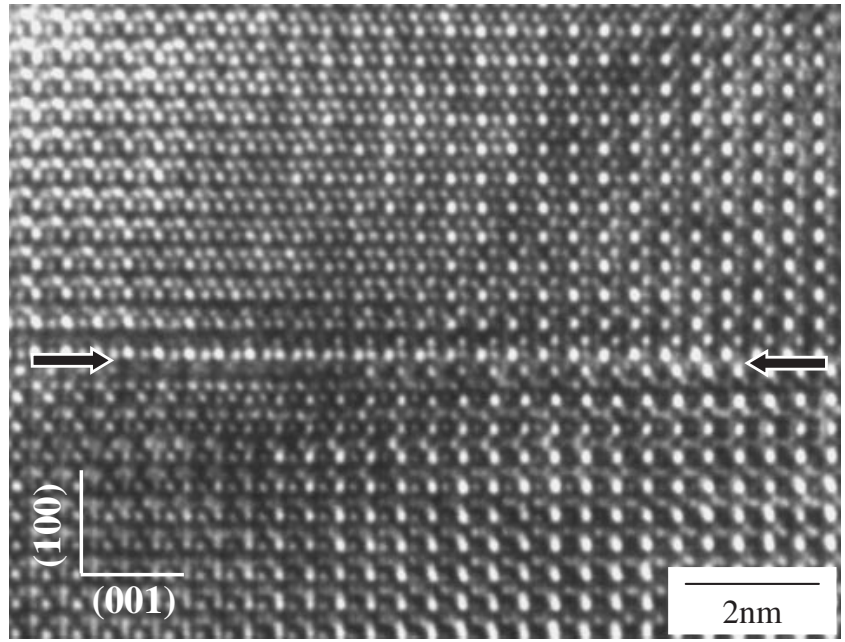

Fig. 4 Two-dimensional lattice image of the APB-like structure along the basal plane in B19 martensite.

transformations is observed by using superlattice reflections for the ordered structure, whereas no contrast is observed using fundamental reflections. ${ }^{7)}$ The APB-like contrast is observed when using both the $10{ }^{*}{ }_{\mathrm{B} 19}$ superlattice and $20{ }^{*}{ }_{\mathrm{B} 19}$ fundamental reflections for the B19 structure, while no contrast is observed using the $002{ }_{\mathrm{B} 19}$ fundamental reflection. This proves that the APB-like contrast does not correspond to the $\pi$ contrast. Therefore, it is likely that the nature of the APB-like contrast is different from that of the APB induced by the order-disorder transformation.

In order to analyze the APB-like contrast at the atomic level, HRTEM observations are carried out from the $[010]_{\mathrm{B} 19}$ zone axis, as shown in Fig. 4. We can identify the interface by the shift of bright spots at the basal plane between the arrows. In other words, the HRTEM image demonstrates that there may be a type of atomic shift along the (001) basal plane corresponding to the APB-like contrast. Therefore, the HAADF-STEM technique through Z-contrast is performed to determine the precise positions of the atomic columns at the interface. Figure 5(a) shows a HAADF-STEM image of the APB-like structure along the basal plane from the $[010]_{\mathrm{B} 19}$ zone axis. Figure 5(b) shows the image intensity profile taken along the white line $\mathrm{X}-\mathrm{Y}$ in Fig. 5(a). The higher intensity profile indicates $\mathrm{Pd}$ columns because of the nature of $\mathrm{Z}$ contrast, while the lower intensity profile indicates $\mathrm{Ti}$ columns. Thus, the bright and dim spots in Fig. 5(a) correspond to the $\mathrm{Pd}$ and $\mathrm{Ti}$ atomic columns, respectively. The interface existing on the basal plane between the arrows in Fig. 5 is the same as that observed in Fig. 4. The Fourier-
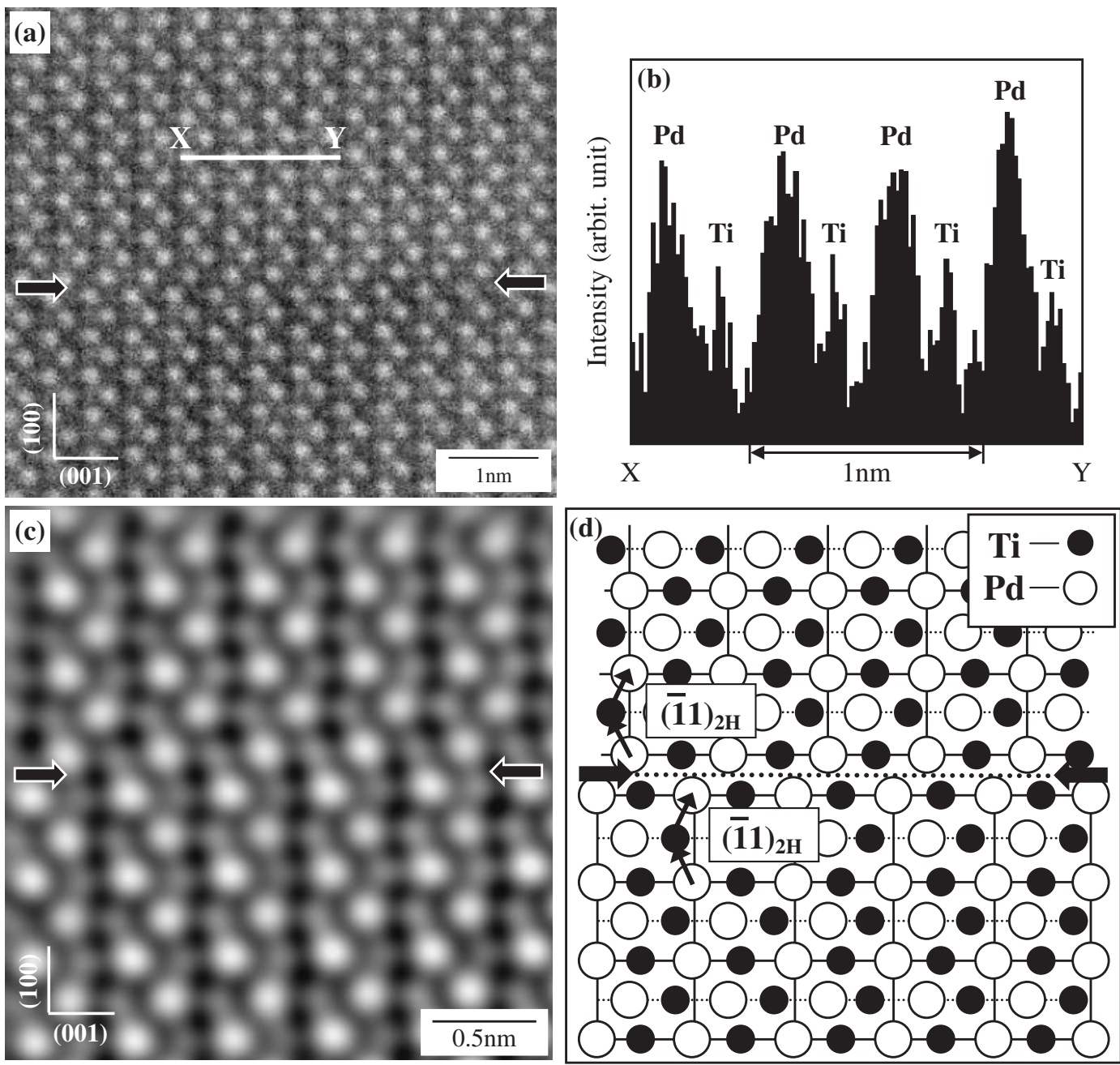

Fig. 5 (a) A HAADF-STEM image of the APB-like structure along the basal plane from the $[010]_{\mathrm{B} 19}$ zone axis. (b) Image intensity profile taken along the white line X-Y in (a). (c) Fourier-filter processed HAADF-STEM image around the APB-like interface. (d) Schematic illustration of the atomic arrangements in (c). 


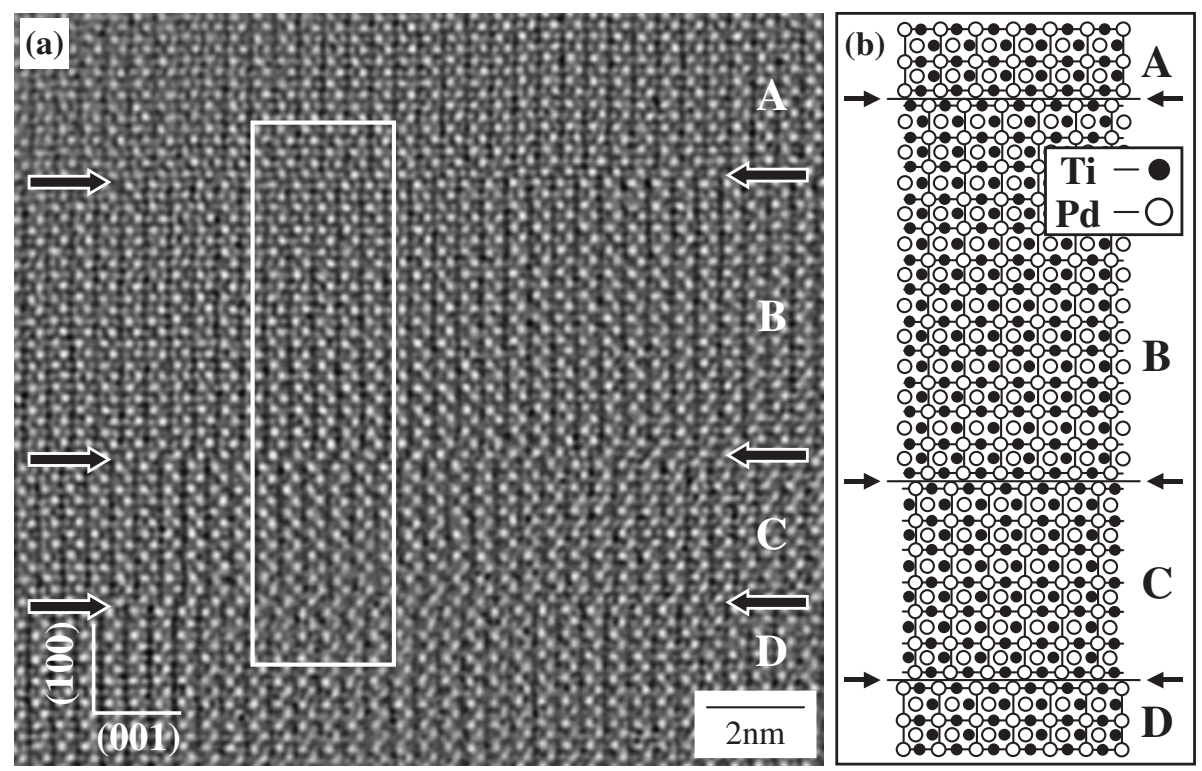

Fig. 6 (a) Fourier-filter processed low-magnification HAADF-STEM image of APB-like structure along the basal plane. (b) Schematic illustration of the atomic arrangements of the white-framed area in (a).

filter processed image around the interface is presented in Fig. 5(c). The atomic arrangements in Fig. 5(c) are schematically illustrated in Fig. 5(d). The open and solid circles indicate $\mathrm{Pd}$ and $\mathrm{Ti}$ atom columns, respectively. There is no doubt that the presence of the atomic shift along the $\boldsymbol{a}$-axis is confirmed across the interface between the arrows. These observations provide a clear evidence of the APB-like structures in B19 martensite. The crystal structure and stacking sequence on both the upper and lower interfaces are $2 \mathrm{H}$ and $(\overline{1} 1)$, respectively, where the expression of $(\overline{1} 1)$ is based on the Zhdanov symbol. ${ }^{8)}$ Here, we emphasize that the interface in the APB-like structure is not defined as a stacking fault since this structure cannot be formed either by the removal or insertion of the atomic plane in the B19 structure. In addition, we have so far reported that the displacement vector of the APB interface in the B2 phase of RS Ti-Pd alloys is $(1 / 2)\langle 111\rangle .{ }^{1)}$ If the APB formed in the B2 parent phase is inherited to the B19 phase after martensitic transformation, the atomic arrangements of the $\mathrm{Ti}$ and $\mathrm{Pd}$ columns on the $(001)_{\mathrm{B} 19}$ and/or $(100)_{\mathrm{B} 19}$ planes should be replaced. However, no irregularity of the atomic arrangements on those planes is recognized, as shown in Fig. 5. Therefore, we conclude that the APB-like structure in the B19 martensite of Ti-Pd alloys is not inherited from the APB in the B2 parent phase, in contrast to the planar defect existing in the martensitic phase of $\mathrm{Cu}$-based alloys. ${ }^{9)}$

Figure 6(a) shows the Fourier-filter processed low-magnification HAADF-STEM image of the APB-like structure. The atomic arrangements of the white-framed area in Fig. 6(a) are schematically illustrated in Fig. 6(b). It is clearly seen that there are four regions from $\mathrm{A}$ to $\mathrm{D}$ in terms of the change in the atomic arrangements between the arrows. The displacement between regions $\mathrm{A}$ and $\mathrm{B}$ is $\langle 1 / 30-1 / 2\rangle$ across the interface. Similarly, the atomic displacement between regions $\mathrm{B}$ and $\mathrm{C}$ is also $\langle 1 / 30-1 / 2\rangle$, and the atomic positions of region $\mathrm{A}$ are the same as those of region D, as illustrated in Fig. 6(b). From these observations, we estimate the displacement vector $\boldsymbol{R}$ as follows: The APB- like contrasts in the present study are observed using $100^{*}{ }_{\mathrm{B} 19}$ superlattice reflections, whereas no contrast is observed by using the $002^{*}{ }_{\mathrm{B} 19}$ fundamental reflection, as mentioned above. In addition, no contrast is observed by using the $010^{*}{ }_{\text {B19 }}$ supperlattice reflection, although we do not reproduce the micrograph here. This fact suggests that there is no atomic displacement along the $\boldsymbol{b}$-axis in the APB-like structure. Therefore, it is concluded that $\boldsymbol{R}$ can be expressed as $\langle 1 / 30-1 / 2\rangle$. The relationship between the phase angle $\boldsymbol{\alpha}$ and $\boldsymbol{R}$ is given by $\boldsymbol{\alpha}=2 \pi \boldsymbol{g} \cdot \boldsymbol{R}{ }^{7)}$ When the value of $\boldsymbol{\alpha}$ is $2 \boldsymbol{n} \pi$, where $\boldsymbol{n}$ is an integer, the fault with $\boldsymbol{R}$ is theoretically invisible. The observations using each $\boldsymbol{g}$ and the proposed $\boldsymbol{R}$ are consistent with this criterion; thus, $\boldsymbol{R}$ of the APB-like structure in the B19 martensite is certainly $\langle 1 / 30-1 / 2\rangle$. The validity of the displacement vector $\boldsymbol{R}$ is considered as follows. The B19 martensitic phase is regarded as a $2 \mathrm{H}$ closepacked layer structure due to the shear and shuffling along the $\pm[1 \overline{1} 0]_{\mathrm{B} 2}$ direction alternately on the $\{110\}_{\mathrm{B} 2}$ plane, ${ }^{8)}$ as illustrated in Fig. 1. In the $2 \mathrm{H}$ structure, triangular nets are formed and the interatomic distance in the layer is all the same, i.e. atoms are arranged centro-symmetric in one layer. Therefore, the atomic displacement along the $\boldsymbol{a}$-axis must be $\pm 1 / 3$ to produce an interface without the breaking of the $2 \mathrm{H}$ structure. Similarly, that along the $c$-axis must be $\pm 1 / 2$, because the $(110)_{\mathrm{B} 2}$ plane corresponds to the $(002)_{\mathrm{B} 19}$ plane, as seen in Fig. 1. Consequently, $\boldsymbol{R}=\langle 1 / 30-1 / 2\rangle$ of the APB-like structure is equal to the displacement due to the atomic shuffling upon the martensitic transformation, since the $2 \mathrm{H}$ structure should be maintained between the interfaces.

Finally, the interface structure of the APB-like structure on a non-basal plane is discussed. Figure 7(a) shows the twodimensional lattice image of the curved APB-like structure in the B19 martensite. It is apparent that there is an atomic shift on the basal plane along the $\boldsymbol{a}$-axis, as indicated by the arrow. Figure 7(b) shows the enlarged micrograph of the framed area in Fig. 7(a). The atomic shift on the non-basal plane as well as that on the basal plane is apparent, as indicated by the arrows. Here, the APB-like structure on the non-basal plane 

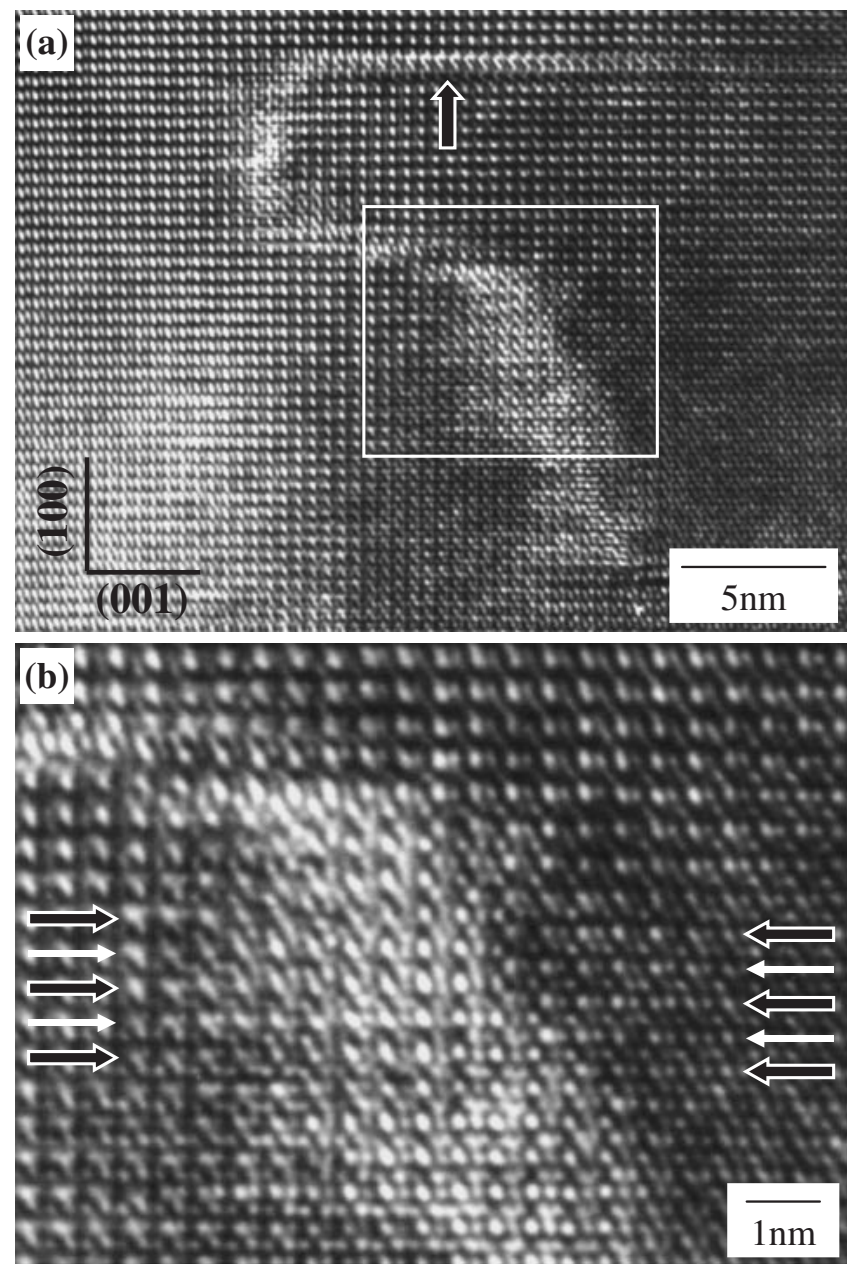

Fig. 7 (a) Two-dimensional lattice image of the curved APB-like structure in B19 martensite. (b) Enlarged micrograph of the framed area in (a).

does not indicate any sharp interface structure, but an interface with a several atomic layers broad since the APBlike structure would consist of two-dimensional interfaces in a three-dimensional crystal, that is, the boundary would be curved in the projected direction.

The formation mechanism of the APB-like structure is proposed as follows on the basis of these observations. During the martensitic transformation from B2 to B19 structures, martensitic domains sporadically nucleate and grow in the B2 parent phase, with each transformed domain eventually coming into contact with each other during the martensitic transformation. The impingement of such domains produces the APB-like interface. Therefore, these facts suggest that the boundary is induced by the local heterogeneity of atomic movements during the martensitic transformation. Consequently, we have newly proposed that the APB-like structure in the B19 martensite is defined as the displacive transformation-induced APB. The formation mechanism of the APB-like structure is still debatable. In order to obtain a clear definition, the support of HAADFSTEM observations throughout the APB-like structure such as closed loop areas on the non-basal plane and in-situ TEM observations before and after the transformation is indispensable. These analyses are now in progress and will be reported in due course.

\section{Concluding Remarks}

An APB-like structure in the B19 martensite of Ti-Pd alloy has been investigated by CTEM, HRTEM, and HAADFSTEM. An APB-like contrast exists along the (001) basal plane of the B19 martensite. It is apparent that the APB-like structure is not inherited from the APB with a displacement vector of the type $\boldsymbol{R}=(1 / 2)\langle 111\rangle$ in the B2 parent phase from HAADF-STEM observations. It is concluded that the displacement vector of the APB-like structure in the B19 martensite can be expressed as $\boldsymbol{R}=\langle 1 / 30-1 / 2\rangle$. On the basis of these observations, the formation mechanism of the APB-like structure is deduced as follows: During the martensitic transformation from B2 to B19 structures, martensitic domains sporadically nucleate and grow in the B2 parent phase, with each transformed domain eventually coming into contact with each other during the martensitic transformation. The impingement of such domains produces the APB interface. These facts suggest that the boundary is induced by the local heterogeneity of atomic movements during the martensitic transformation. Therefore, we have newly proposed that the APB-like structure in the B19 martensite is defined as the displacive transformationinduced APB.

\section{Acknowledgments}

This work was supported by a "Grant-in-Aid for Scientific Research (B)" from the Japanese Society for the Promotion of Science (JSPS)," “Grant-in-Aid for Young Scientists (B)" and "Nanotechnology Network Project" of the Ministry of Education, Culture, Sports, Science and Technology (MEXT), Japan. The authors would like to express their sincere appreciation to Dr. K. Kimoto and Dr. M. Saito of the National Institute for Materials Science for their support in the HAADF-STEM experiments and Prof. R. Tomoshige and Dr. S. Ii of Sojo University for their support in the HRTEM experiments. The authors are most grateful to Prof. M. Itakura of Kyushu University for their valuable comments. The authors also thank Mr. M. Yamashita for his assistance in the preparation of TEM specimens.

\section{REFERENCES}

1) M. Matsuda, T. Hara, E. Okunishi and M. Nishida: Philos. Mag. Lett. 87 (2007) 59-64.

2) H. C. Donkersloot and J. H. N. Van Vucht: J. Less-Comm. Met. 20 (1970) 83-91.

3) K. Otsuka and X. Ren: Prog. Mater. Sci. 50 (2005) 511-678.

4) A. E. Dwight, R. A. Conner and J. W. Downey: Acta Cryst. 18 (1965) 835-839.

5) M. Nishida, T. Hara, Y. Morizono, A. Ikeya, H. Kijima and A. Chiba: Acta Mater. 45 (1997) 4847-4853.

6) Y. Shugo and T. Honma: Bull. Res. Inst. Miner. Dressing Metall. Tohoku Univ. 43 (1987) 117-127.

7) G. Thomas and J. Washburn: Electron Microscopy and Strength of Crystals, (Interscience, New York, 1963) pp. 333-440.

8) Z. Nishiyama: Martensitic Transformation, (Academic Press, New York, 1978).

9) J. W. Seo and D. Schryvers: Acta Mater. 46 (1998) 1177-1183. 